Artículos científicos

\title{
La inclusión de niños sordos en educación básica en una escuela de México mediante el diseño de recursos digitales
}

\author{
Inclusive education for deaf children in a Mexican Elementary school \\ through digital tools design
}

\section{A inclusão de crianças surdas na educação básica em uma escola no México por meio do design de recursos digitais}

\author{
Paloma Trejo Muñoz \\ Universidad Autónoma de Querétaro, México \\ paloma.moodle@gmail.com \\ https://orcid.org/0000-0001-9567-9674 \\ Sandra Martínez Pérez \\ Universidad de Barcelona, España \\ smartinezperez8@gmail.com \\ https://orcid.org/0000-0002-7458-1077
}

\section{Resumen}

La gran variedad de situaciones a las que se enfrentan las personas sordas desde el nacimiento las ha llevado a diferentes tipos de escolarización. El sistema educativo mexicano ha generado diversos procesos de inclusión educativa sustentados en los derechos de los individuos. Entre las ofertas educativas a nivel básico, en el estado de Querétaro, en la extensión del Instituto Queretano Marista (EIQM), se ha iniciado un estilo de inclusión a través de la lengua de señas mexicana (LSM) y la enseñanza de español.

El objetivo de esta investigación fue diseñar y aplicar una herramienta digital para la enseñanza del español de los alumnos sordos en educación básica. El trabajo se realizó en cuatro fases utilizando la investigación basada en el diseño (IBD). La primera fase estuvo enfocada al análisis de problemas, la segunda consistió en el desarrollo de soluciones e 

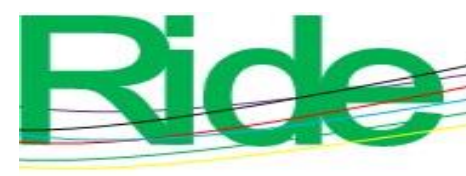

Revista Iberoamericana para la Investigación y el Desarrollo Educativo

ISSN 2007 - 7467

innovaciones tecnológicas que pudieran favorecer la enseñanza de español, la tercera consistió en la aplicación de las soluciones de forma iterativa (es decir, a modo de prueba y error) y la última fase permitió enmarcar los principios de diseño acordes a las necesidades del alumnado. El desarrollo de los recursos digitales incluyó la realización de animaciones 2D, videos y juegos. El equipo de profesionales estuvo conformado por intérpretes educativos, personas sordas y profesores de grado.

Los primeros resultados obtenidos demuestran cómo las herramientas digitales son complementos que se aplican en el aula para favorecer los procesos de aprendizaje del alumnado sordo y un acompañamiento a profesores y tutores para la enseñanza de la lengua española. La investigación expone que las herramientas digitales basadas en el juego, recursos visuales y kinestésicos, así como la evaluación procesual favorecen la atención y la repetición para la comprensión de la segunda lengua en alumnos sordos. La investigación demuestra que no solo la tecnología, sino también la cultura pueden ofrecer oportunidades para el aprendizaje de las personas sordas.

Palabras clave: educación básica, inclusión, investigación basada en el diseño, niños sordos, recursos digitales.

\section{Abstract}

The wide variety of life issues that deaf people face from birth has led them to different types of schooling. The Mexican Educational System has made different processes of inclusive education based on individuals' rights. Among the educational offerings at elementary school, in the State of Querétaro, specifically at the Extensión del Instituto Queretano Marista (EIQM), an inclusive education type has been introduced through the Mexican Sign Language (LSM) and the teaching of Spanish language.

The research objective was to design and apply a digital tool for teaching Spanish to deaf students in Elementary School. The research was conducted in four phases using Design Based Research (IBD). The first phase focused on problem analysis, the second phase consisted of developing solutions and technological innovations that could favor the teaching of Spanish, the third phase was based on the application of solutions in an iterative way, that is, on an experimental basis, the fourth phase allowed framing the design principles according to the needs of the students. The development of digital resources included making 2D 


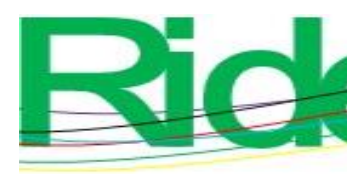

\section{Revista Iberoamericana para la Investigación y el Desarrollo Educativo ISSN 2007 - 7467}

animations, videos, and games. The team of professionals included educational interpreters, deaf people and teachers.

Digital tools are not the whole of teaching, but a classroom complement and a supplement for teachers and tutors. The research shows that digital game-based tools, visual and kinesthetic resources, as well as procedural evaluation favor attention and repetition for the understanding of the second language in deaf students. Research shows that not only technology, but culture can expand learning opportunities for deaf people.

Keywords: elementary school, inclusive education, design based reseach, deaf children, digital tools.

\section{Resumo}

A grande variedade de situações que os surdos enfrentam desde o nascimento os leva a diferentes tipos de escolaridade. O sistema educacional mexicano gerou diversos processos de inclusão educacional baseados nos direitos das pessoas. Entre as ofertas educacionais de nível básico, no estado de Querétaro, na extensão do Instituto Queretano Marista (EIQM), iniciou-se um estilo de inclusão por meio da língua de sinais mexicana (LSM) e do ensino do espanhol.

O objetivo desta pesquisa foi projetar e aplicar uma ferramenta digital para o ensino de espanhol para alunos surdos na educação básica. $\mathrm{O}$ trabalho foi realizado em quatro fases usando pesquisa baseada em design (IBD). A primeira fase foi focada na análise de problemas, a segunda consistiu no desenvolvimento de soluções e inovações tecnológicas que pudessem favorecer o ensino do espanhol, a terceira consistiu na aplicação das soluções de forma iterativa (ou seja, como um teste e erro) e a última fase permitiu enquadrar os princípios de design de acordo com as necessidades dos alunos. O desenvolvimento de recursos digitais incluiu a realização de animações $2 \mathrm{D}$, vídeos e jogos. A equipe de profissionais era composta por intérpretes pedagógicos, surdos e professores universitários. Os primeiros resultados obtidos demonstram como as ferramentas digitais são complementos que se aplicam em sala de aula para favorecer os processos de aprendizagem de alunos surdos e apoiar professores e tutores para o ensino da língua espanhola. A pesquisa mostra que ferramentas digitais baseadas em recursos lúdicos, visuais e cinestésicos, bem como avaliação processual, favorecem a atenção e a repetição para a compreensão da segunda 


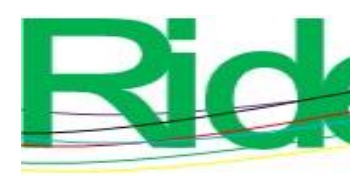

Revista Iberoamericana para la Investigación y el Desarrollo Educativo

ISSN 2007 - 7467

língua em alunos surdos. A pesquisa mostra que não apenas a tecnologia, mas também a cultura podem fornecer oportunidades de aprendizagem para pessoas surdas.

Palavras-chave: educação básica, inclusão, pesquisa baseada em design, crianças surdas, recursos digitais.

Fecha Recepción: Mayo 2020

Fecha Aceptación: Octubre 2020

\section{Introducción}

La interacción humana con el mundo inicia desde el nacimiento. Los sentidos ayudan a descubrir los olores, las texturas, los sabores, los colores y los sonidos. La carencia de cualquiera de estos implica una limitante para descubrir el exterior. La persona declarada sorda puede tener (o no) restos auditivos, haber perdido la audición en algún momento de su vida o haber nacido sin la posibilidad de oír. La familia — dependiendo de los conocimientos que tenga sobre la temática, el asesoramiento por parte de los diversos profesionales, de su posición económica, social y cultural - determinará el momento y tipo de escolarización que recibirán sus hijos: escuelas tradicionales, centros de atención múltiple (CAM), colegios inclusivos o educación en casa (Brunot, 2019; García, 2018; Romero y García, 2013).

En México, la Secretaría de Educación Pública (SEP, 2015, 2016, 2017) ha invitado a las instituciones públicas y privadas a generar procesos educativos que promuevan la inclusión de las personas con diversidad funcional. En este sentido, las nuevas directrices apuntan, por un lado, hacia una metamorfosis en las estructuras del sistema educativo, que pretenden poner la atención en el cómo, el qué y el para qué de la educación, con énfasis en que la formación es un elemento clave no solo para el alumnado, sino también para el profesorado. Y por otro, generar prácticas inclusivas por parte de los docentes de las escuelas "normalistas", promoviendo la participación y colaboración de toda la comunidad educativa. Con esto se procura entender que "la diversidad da la oportunidad para aprender y enseñar con ella, y garantiza el acceso, la permanencia, la participación y el progreso de todos los estudiantes" (Bonilla, Fernández y Vázquez, 2019, p. 5). Explicado lo anterior, se puede decir que la presente investigación ha sido desarrollada en la extensión del Instituto Queretano Marista (EIQM), que ha iniciado un programa de inclusión, adoptando como postura la existencia de una comunidad de personas sordas, la aceptación de la lengua de señas mexicana (LSM) como primera lengua (L1), el español como segunda lengua (L2) y la existencia de un puente de comunicación entre oyentes (las personas que pueden oír) y 


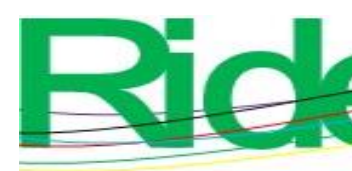

\section{Revista Iberoamericana para la Investigación y el Desarrollo Educativo ISSN 2007 - 7467}

sordos (cualquier persona con diversidad funcional auditiva): los intérpretes educativos de LSM. Para ello, bajo la perspectiva de la investigación basada en el diseño (IBD), se han elaborado unos recursos digitales con la finalidad de favorecer y potenciar la enseñanza de la segunda lengua: el español.

La trascendencia de la investigación residió en el reconocimiento de un colectivo de personas que ha sido clasificado en el ámbito de la discapacidad, un término políticamente aceptado en México para hacer referencia a las personas con alguna situación física diferente a lo común, y no como individuos pertenecientes a un colectivo cultural que demanda respeto por sus derechos, manifestaciones culturales, espacios, así como manera de aprender, entre otros (Lissi, Svartholm y González, 2012; Moores, 1990). En consecuencia, se pone de facto la urgencia de que la sociedad, a través de la búsqueda de recursos e investigaciones, coadyuve a la creación de espacios y desarrollo de recursos y materiales para promover el desarrollo pleno de todas las personas (Armstrong, Armstrong y Spandagou, 2010; García, 2018; Lankshear y Knobel, 2008).

Para el desarrollo de la herramienta digital se distinguieron técnicas efectivas para la enseñanza del español (L2), las cuales deberían favorecer las clases presenciales en el colegio. Para ello, se analizaron diversas estrategias de enseñanza, desde el tipo de alumno que se recibe hasta las características de los profesores que atienden a este colectivo; para identificar cómo las técnicas de enseñanza llevadas a cabo para la enseñanza del estudiante sordo permiten el uso de tecnología para apoyar la instrucción de una L2, así como también los espacios y tiempos apropiados para el desarrollo de la herramienta y la consecución del objetivo planteado.

\section{La inclusión de la comunidad sorda de Querétaro: de la L1 a la L2}

La comunidad sorda del estado de Querétaro ha desplegado sus propios espacios de encuentro y participación. En estos, los jóvenes han enfocado sus esfuerzos para desenvolverse artísticamente en el ámbito de la ilustración y la pintura, así como en equipos deportivos, especialmente en el soccer, y como profesionistas en terapia física, animación y administración. No obstante, la inclusión para personas sordas ha tenido diversas dificultades, como la falta de formación y sensibilización del profesorado. Son pocas las personas interesadas en el aprendizaje, aplicación e interpretación de la LSM y, por consiguiente, en la comunicación. 


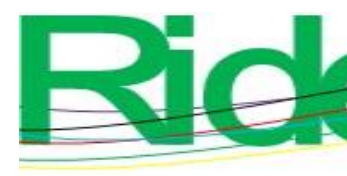

Revista Iberoamericana para la Investigación y el Desarrollo Educativo ISSN 2007 - 7467

Desde el enfoque inclusivo, es necesario que las instituciones educativas, como afirman Booth y Ainscow (2002), tengan en cuenta tres dimensiones: las culturas inclusivas, las políticas inclusivas y las prácticas inclusivas. De este modo, la inclusión se visualiza como igualdad de oportunidades para todas las personas, a partir de la accesibilidad, buscando romper todo tipo de barreras a partir de modelos inclusivos y democráticos (Unicef, 2017).

El desarrollo de una L1 es fundamental para el aprendizaje de una L2 (Navarro, 2010). A las personas sordas se les reconoce el desarrollo de la LSM (L1), pero no la L2 (el español). El modelo de inclusión implementado en el colegio tiene su razón de ser en las necesidades observadas para promover la continuidad de los estudios, por lo que se adoptó el modelo de enseñanza de L2 y la reflexión de la LSM como L1, impulsando a que, al término de la educación básica, los estudiantes sordos realicen el examen de certificación correspondiente y accedan a grados académicos posteriores con las competencias lingüísticas necesarias e indispensables para comprender, analizar y cuestionar, en su caso, los contenidos.

La comunicación es una habilidad que les permite a los seres humanos involucrarse, participar e interactuar en la sociedad desde sus primeros meses de vida; igualmente, les da la oportunidad de comprender su relación con el entorno en el que se desenvuelven a través de procesos fundamentales que requieren de percepción, memoria, categorización y procesamiento de la información (Lieberman, Hatrak y Mayberry, 2014). Esta oportunidad de aprender una forma de comunicación natural atendiendo a toda la complejidad sintáctica es crucial para el desarrollo del cerebro, la organización de las ideas y, por tanto, para la adquisición de otras lenguas en el transcurso de la vida (Skotara, Salden, Kügow, HänelFaulhaber y Röder, 2012). Dicha forma de comunicación natural únicamente se puede aprender desde los primeros años si es accesible, interactiva y abundante (Gertz y Boudreault, 2016). Así pues, el aprendizaje de una lengua es un proceso activo, en el que se descubren sus reglas y conceptos a través de una manipulación creativa y pruebas de hipótesis. La diversidad de la experiencia lingüística en cuanto a la recepción de información y la expresión es necesaria para la adquisición y el desarrollo del lenguaje. Un niño es un agente activo en su propio desarrollo lingüístico (Brennan, 1975). 


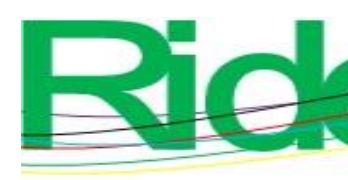

Revista Iberoamericana para la Investigación y el Desarrollo Educativo

ISSN 2007 - 7467

Lo anterior invita a plantearse cuestionamientos en el ámbito de la educación como el siguiente: ¿se está logrando que las personas sordas adquieran una lengua que les permita tener acceso a su desarrollo cognitivo? Aproximadamente $90 \%$ de los infantes sordos nacen de padres oyentes (Cole y Flexer, 2019; Mitchell, 2004; Moores, 1990), lo que implica nuevos desafíos en el proceso de enseñanza de una lengua: el primero es cómo hacer para que una persona que no escucha entienda o comprenda lo que se le expresa; el segundo, qué hacer para minimizar el problema, a qué especialistas o instituciones acudir para conjuntar esfuerzos y procesos.

De acuerdo con los estudios realizados por Goldin-Meadow y Mayberry (2001), si los infantes sordos nacen en familias donde los padres son oyentes, se necesita intervenir de maneras diversas. La detección temprana de la pérdida auditiva debe estar acompañada de la entrada pronta a un sistema educativo donde se enseñe la LSM y estar en contacto con personas que la hablen de manera fluida, es decir, asegurarse que el infante sordo tenga acceso a la lengua de señas desde pequeño (Hurford, 1991; Stokoe, 2005). Por otro lado, Susskind y Susskind (2017) explican que a la edad de tres años y medio el cerebro humano ha completado $85 \%$ de su crecimiento, por lo que estos primeros años de vida son los cruciales en el desarrollo de la lengua. Así pues, una persona sorda que no tenga en los primeros años de vida acceso al lenguaje será semilingüe. El término semilingüismo es utilizado, tal y como exponen Fridman (2009), para explicar que una persona puede hablar dos lenguas, pero no las domina totalmente, es decir, el dominio de una lengua es mayor que la otra. En este caso, las personas sordas tienen un mayor dominio de la LSM que de la lengua española.

La adquisición de la LSM, como L1, permite a los alumnos sordos comunicarse efectivamente entre ellos, posibilitando que en la sala de clases se pueda dar una conversación colectiva entre docente y alumnado, y no solo un diálogo uno a uno entre alumno y profesor. Esta fluidez en la comunicación, favorecida con un intérprete educativo de LSM en el aula, permite también la realización de actividades de trabajo colaborativo, las que a su vez potencian no solo el aprendizaje de los contenidos, sino también el desarrollo lingüístico de la L1 y la L2, dado que los alumnos presentan diferentes niveles de desempeño en una y otra lengua (Lissi, Svartholm y González, 2012). 


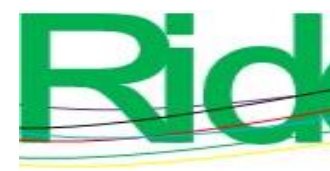

Revista Iberoamericana para la Investigación y el Desarrollo Educativo ISSN 2007 - 7467

Es sustancial remarcar que la LSM no es una traducción del español y que además es ágrafa, por lo que el uso de tecnología de subtítulos en pantalla no resuelve la fluidez en la comunicación dentro del aula. Por eso, el dominio de la LSM por parte del alumno es fundamental para comprender al profesor a través del intérprete educativo.

Todos estos factores son determinantes para decidir cómo será enseñada la L2 en un ambiente de inclusión. Una de las condiciones para iniciar el proceso de inclusión —además de la aceptación de la LSM como L1 - es la adopción de la LSM como parte de un proceso cultural de toda la escuela, es decir, como una materia extra para los grupos en los que hay infantes sordos.

Respecto al aprendizaje de las lenguas en el currículo, se presenta una diferencia entre oyentes y sordos como se muestra en la tabla de clases de lenguas para sordos y oyentes.

Tabla 1. Clases de lenguas para sordos y oyentes

\begin{tabular}{|c|c|c|}
\hline & Lengua materna & Lengua extranjera \\
\hline Oyentes & Reflexión de la lengua española & Aprendizaje de la lengua inglesa \\
\hline Sordos & Reflexión de la lengua de señas & Aprendizaje de la lengua española \\
\hline
\end{tabular}

Entre los desafíos que se presentan para la educación e instrucción de las personas sordas se encuentra la falta de estandarización de la LSM, es decir, existen pocos estudios sobre esta, y quienes la utilizan generalmente aprenden de manera empírica al acercarse a las comunidades sordas. Esto no es una limitante, aunque es más sencillo enseñar una L2 si se ha reflexionado en la estructura de la L1.

La educación bilingüe bicultural acepta el uso de dos lenguas de manera diferenciada, esto es, el profesor hablando español mientras un intérprete educativo transmite la información en LSM. Gracias a esta educación es posible potenciar el desarrollo de la LSM como L1 y a su vez utilizarla para facilitar el aprendizaje del español escrito. Este tipo de educación necesita un ambiente en el que se enfatice — además de la LSM — la formación de profesores oyentes y sordos para que los estudiantes se vuelvan usuarios competentes de las dos lenguas (Lissi, Svartholm y González, 2012), ya que en la medida en que dominen su lengua, será más sencillo introducir la L2.

Para poder enseñar una L2 a personas con diversidad funcional auditiva, dentro de la presente investigación, se determinaron condiciones para resolver, como son los cimientos 


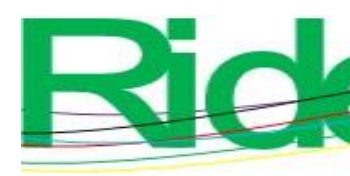

Revista Iberoamericana para la Investigación y el Desarrollo Educativo

ISSN 2007 - 7467

de una L1 y los procedimientos para poder llevar de forma simultánea la enseñanza académica en su L2.

\section{Contexto de la investigación}

La investigación se desarrolló en la extensión del Instituto Queretano Marista (EIQM), institución que busca educar a niños y jóvenes desde una educación humanizada y personal (Ruiz de Chávez et al., 2013) que da respuesta a personas con discapacidad, diseñando y creando programas provinciales, entre ellos el llamado Grupo Especial Marista (GEM). Este procura ayudar al alumnado a su madurez personal, lograr su independencia, reconocer su dignidad, así como brindar espacios para socializar y sentirse aceptados. Además, dicha institución también planificó su propio sistema de inclusión para estudiantes sordos y lo puso a disposición de este proyecto de investigación.

El sistema de inclusión busca que las personas sordas adquieran naturalmente la LSM y a partir de esta aprendan la L2, todo esto a través del trabajo del profesorado de grado y de talleres, y los intérpretes educativos de LSM. El objetivo es procurar que los niños puedan ser incluidos con su intérprete en un grupo regular desde tercero o cuarto de primaria.

El proyecto para estudiantes sordos no es un programa provincial; sin embargo, la experiencia observada con los grupos GEM y el trabajo desarrollado en la Universidad Marista, campus Ciudad de México — que ya ha dado servicio a una persona sorda de nacimiento, graduada en 2013 como psicóloga — habla de una experiencia comprobable que aporta en mucho al proyecto de investigación.

Este nuevo proyecto ha sido apoyado con una herramienta digital. La tecnología ha demostrado ser útil para hacer eficientes los procesos de alfabetización. Los ambientes de aprendizaje se ven favorecidos por su uso, de una manera accesible, estimulando la creatividad y la innovación en los usuarios. Los estudiantes pueden discutir sus ideas a lo largo del proceso de escritura a través de formas síncronas y asíncronas. Un aspecto importante de la alfabetización digital es que permite tanto a estudiantes como a profesores usar diferentes medios (Lankshear y Knobel, 2008).

Los entornos digitales pueden fomentar la instrucción académica de los estudiantes sordos en el aula, haciendo posible el procesamiento de textos, conferencias grupales, envío y recepción de correo electrónico, almacenamiento y análisis de datos (Cacheiro, Sánchez y 


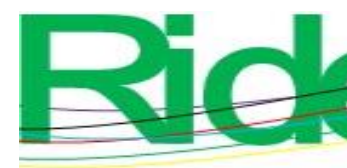

Revista lberoamericana para la Investigación y el Desarrollo Educativo

ISSN $2007-7467$

González, 2016; Martínez, Gutiérrez y Fernández, 2018). La tecnología permite que los alumnos y profesores puedan compartir pensamientos e ideas expresadas en texto.

\section{Metodología}

El presente trabajo fue de naturaleza cualitativa y la técnica empleada fue la investigación basada en el diseño (IBD). El objetivo de esta fue diseñar y aplicar una herramienta digital para la enseñanza del español de los alumnos sordos en educación básica de la EIQM. En virtud de las condiciones en las que se emplea este tipo de investigación, debía permitir realizar el proyecto inclusivo desde el primer grado de primaria y el acceso a realizar cambios en el proceso, partiendo de que la IBD pretende dar solución a un problema práctico y complejo. La investigación se describe como adaptativa, colaborativa, contextual, flexible, orientada a objetivos, aterrizada, integrativa, interactiva, intervencionista, metodológicamente inclusiva, multinivel, pragmática, enfocada en el proceso, teórica, transformativa y orientada a la utilidad (Cobb, Confrey, DiSessa, Lehrer y Schauble, 2003; Gros, 2007; Kelly, 2003; McKenney y Reeves, 2012; Mckenney, van den Akker y Nieveen, 2006; Wang y Hannafin, 2005). De este tipo de investigación pueden resultar contribuciones teóricas o prácticas para ser replicadas en diferentes contextos.

Las técnicas para la obtención de los datos fueron la entrevista semiestructurada, la observación participante y el análisis documental. La información fue examinada de manera cualitativa, analizando contenidos, inducción analítica y su triangulación. Para las entrevistas se utilizó un guión determinado (Bisquerra, 2016) para identificar la información relevante. Las entrevistas se aplicaron a docentes, padres de familia y a los nuevos estudiantes sordos.

Igualmente, se empleó el modelo propuesto por Reeves (2006) para implementar la IBD (tabla 2), considerando el ámbito tecnológico de la investigación. 
Tabla 2. Fases de la IBD

\begin{tabular}{|c|c|c|c|}
\hline FASE 1 & FASE 2 & FASE 3 & FASE 4 \\
\hline Análisis de & Desarrollo de & Ciclos iterativos de & Reflexión \\
\hline problemas prácticos & soluciones basadas & pruebas y mejoras & $\begin{array}{ll}\text { desarrollar los } & \text { los } \\
\text { princinios de diseñ }\end{array}$ \\
\hline $\begin{array}{lr}\text { a traves } & \text { de } \\
\text { investigadores } & y\end{array}$ & $\begin{array}{l}\text { en los principios de } \\
\text { diseño existentes } y\end{array}$ & $\begin{array}{l}\text { de soluciones en la } \\
\text { práctica. }\end{array}$ & $\begin{array}{l}\text { principios de diseno } \\
y \quad \text { mejorar la }\end{array}$ \\
\hline $\begin{array}{l}\text { practicantes en } \\
\text { colaboración. }\end{array}$ & $\begin{array}{l}\text { las innovaciones } \\
\text { tecnológicas. }\end{array}$ & & $\begin{array}{l}\text { implementación de } \\
\text { la solución. }\end{array}$ \\
\hline
\end{tabular}

Fuente: Reeves (2006, p. 59)

Según el enfoque de la IBD, se pueden observar diferentes implicaciones tanto en el diseño como en la exploración de innovaciones educativas. A su vez, también requiere de una implicación a nivel didáctico, en este caso de docentes e intérpretes; organizativo, la involucración de la dirección y la mejora de los planteamientos pedagógicos; y los posibles recursos, entre ellos los digitales, como núcleos de esas innovaciones y contribuyendo, consecuentemente, a una mejor comprensión de la naturaleza y condiciones de aprendizaje (Bell, Hoadley y Linn, 2004; Gibelli, 2014).

En la tabla 3 se pueden observar las diferentes fases de la investigación con sus correspondientes participantes y actividades.

Tabla 3. Fases de la IBD de la investigación

\begin{tabular}{|c|c|c|c|}
\hline FASES & Descripción & Participantes & Actividades \\
\hline Fase 1 & $\begin{array}{l}\text { Análisis } \\
\text { problema. }\end{array}$ & $\begin{array}{l}\text { Investigador, padres de } \\
\text { familia, profesores, } \\
\text { intérpretes, alumnado }\end{array}$ & $\begin{array}{l}\text { Entrevistas semiestructuradas, } \\
\text { observación no participante y } \\
\text { pruebas diagnóstica de habilidades } \\
\text { cognitivas. }\end{array}$ \\
\hline Fase 2 & $\begin{array}{l}\text { Desarrollo de } \\
\text { herramientas } \\
\text { digitales. }\end{array}$ & $\begin{array}{l}\text { Investigador } \\
\text { profesionales. }\end{array}$ & $\begin{array}{l}\text { Trabajo con expertos, revisión de } \\
\text { de herramientas educativas, } \\
\text { búsqueda de tecnología accesible. } \\
\text { Desarrollo de herramienta. }\end{array}$ \\
\hline Fase 3 & $\begin{array}{l}\text { Evaluación de } \\
\text { soluciones sobre la } \\
\text { marcha. }\end{array}$ & $\begin{array}{l}\text { Con los involucrados y } \\
\text { estudiantes sordos. } \\
\text { Evaluación de los } \\
\text { expertos. }\end{array}$ & $\begin{array}{l}\text { Iteraciones con la herramienta, } \\
\text { revisión de resultados. }\end{array}$ \\
\hline Fase 4 & $\begin{array}{lr}\text { Documentación } & \text { y } \\
\text { reflexión } & \text { para } \\
\text { introducir } & \text { los } \\
\text { principios de diseño }\end{array}$ & $\begin{array}{l}\text { Investigador } \\
\text { profesionales. }\end{array}$ & $\begin{array}{l}\text { Elaboración de principios para el } \\
\text { diseño de la herramienta, mejora de } \\
\text { soluciones e implementación. }\end{array}$ \\
\hline
\end{tabular}

Fuente: Elaboración propia 
En la fase 1, las entrevistas semiestructuradas a padres de familia permitieron conocer a) la procedencia académica de los niños; b) la situación familiar del alumnado; c) las perspectivas académicas de los padres de familia sobre sus hijos; d) la escolaridad de los familiares, y e) otros aspectos relevantes.

Tabla 4. Participantes

\begin{tabular}{|c|c|c|c|}
\hline Participante & GÉNERO & EDAD & $\begin{array}{c}\text { USUARIO DE } \\
\text { LSM }\end{array}$ \\
\hline Infante sordo & $\mathrm{M}$ & 8 & Sí \\
\hline Infante sordo & $\mathrm{F}$ & 8 & Sí \\
\hline Infante sordo & $\mathrm{F}$ & 8 & Sí \\
\hline Infante sordo & $\mathrm{F}$ & 8 & Sí \\
\hline Infante sordo & $\mathrm{F}$ & 7 & Sí \\
\hline Infante sordo & $\mathrm{M}$ & 7 & Sí \\
\hline Infante sordo & $\mathrm{M}$ & 7 & Sí \\
\hline Infante sordo & $\mathrm{M}$ & 7 & No \\
\hline Adulto padre familia & $\mathrm{F}$ & 42 & Sí \\
\hline Adulto padre familia & $\mathrm{F}$ & 38 & No \\
\hline Adulto padre familia & $\mathrm{M}$ & 36 & No \\
\hline Adulto padre familia & $\mathrm{M}$ & 34 & No \\
\hline Adulto padre familia & $\mathrm{F}$ & 38 & No \\
\hline Intérprete & $\mathrm{F}$ & 45 & No \\
\hline Intérprete & $\mathrm{F}$ & 36 & No \\
\hline Administrativo & $\mathrm{F}$ & 55 & 42 \\
\hline Administrativo & $\mathrm{F}$ & & \\
\hline
\end{tabular}

Fuente: Elaboración propia

En lo que respecta a las entrevistas a intérpretes y administración del colegio, se pudo conocer a) el nivel académico con el que se recibieron los niños; b) las expectativas de aprendizaje; c) las necesidades de aprendizaje, y d) el modelo de inclusión llevado a cabo en el colegio.

La segunda fase estuvo dirigida al desarrollo de soluciones informadas a través de principios de diseño existentes e innovaciones tecnológicas. Para el desarrollo de esta fase se precisó conocer el Modelo Educativo Marista (MEM), el cual contiene elementos de diseño instruccional, determinación del currículo de la enseñanza del español como L2 y los materiales orientados a ello, así como las expectativas de los intérpretes y profesores. El MEM especifica que su patrón de planeación se enfoca en el desarrollo sociocognitivo del estudiante. En cuanto al diseño instruccional, se examinó la literatura sobre la enseñanza de español como L2 y sus limitaciones para estudiantes sordos. Se auxilió al colegio en la búsqueda de un texto que acompañara a los estudiantes en los aprendizajes esperados del 


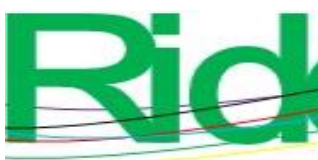

\section{Revista Iberoamericana para la Investigación y el Desarrollo Educativo ISSN 2007 - 7467}

primer al sexto grado de primaria, y se elaboró con ellos los estándares esperados con fundamento en el marco común europeo de referencia para las lenguas (MCERL), en el ajuste de los aprendizajes indicados en el MEM, así como en el modelo de planificación didáctica (tabla 5) (Ruiz de Chávez et al., 2016).

Tabla 5. Modelo de planeación corta del modelo

\begin{tabular}{|c|c|c|}
\hline \multicolumn{2}{|c|}{ Etapa } & $\begin{array}{l}\text { Actividades como proceso de aprendizaje } \\
\begin{array}{l}\text { Destreza + contenido + técnica de aprendizaje + actitud } \\
d+c+t+a\end{array}\end{array}$ \\
\hline \multicolumn{3}{|r|}{ Capacidades-destrezas } \\
\hline \multirow{5}{*}{ Contexto } & Inicio & Actividad 1: $d+c+t+a$ \\
\hline & Desarrollo & Actividad 2: $\mathrm{d}+\mathrm{c}+\mathrm{t}+\mathrm{a}$ \\
\hline & & Actividad $\mathrm{n}: \mathrm{d}+\mathrm{c}+\mathrm{t}+\mathrm{a}$ \\
\hline & Cierre & Actividad $\mathrm{n}: \mathrm{d}+\mathrm{c}+\mathrm{t}+\mathrm{a}$ \\
\hline & MSA & Actividad $\mathrm{n}: \mathrm{d}+\mathrm{c}+\mathrm{t}+\mathrm{a}$ \\
\hline
\end{tabular}

Fuente: Ruiz de Chávez et al. (2016)

A partir de este modelo, se elaboraron las planeaciones por unidad temática. La tabla 6 muestra el formato utilizado para cada unidad. Cada planeación debe mostrar las capacidades-destrezas, valores-actitudes, así como los contenidos y técnicas de aprendizaje.

Tabla 6. Planeación con el modelo

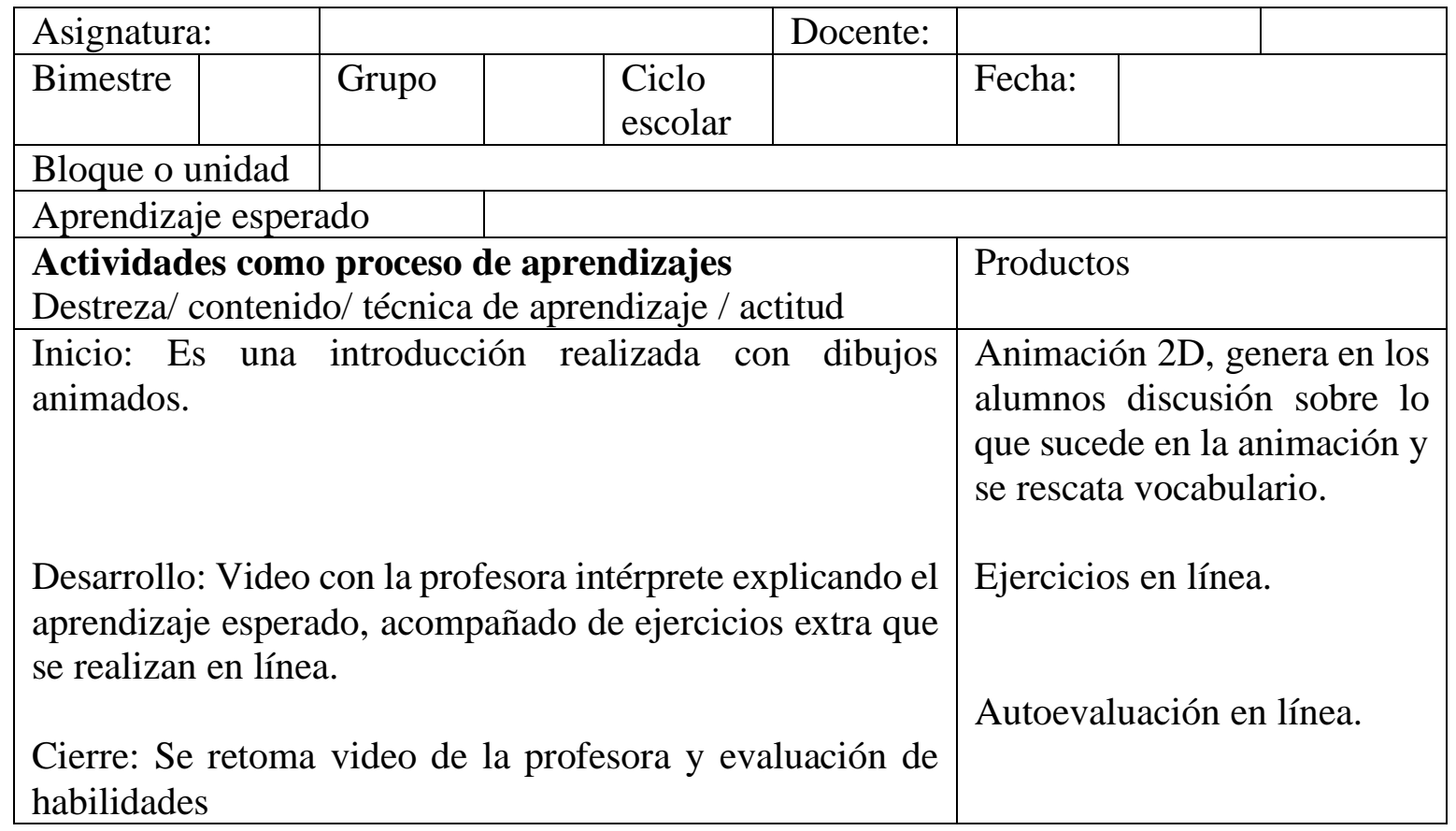

Fuente: Elaboración propia 

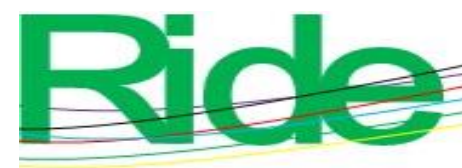

Revista Iberoamericana para la Investigación y el Desarrollo Educativo

ISSN 2007 - 7467

Al inicio de cada sesión se puede visualizar un video 2D. Para la elaboración de estos se entregó el listado del nuevo vocabulario a una dibujante sorda, con base en sus experiencias, y teniendo en cuenta el objetivo de la investigación creó la trama de cada uno para que los contenidos fueran relevantes para el alumnado. Las animaciones son una secuencia de cuentos donde el personaje principal es una niña sorda que se acaba de integrar al colegio. Los dibujos animados no tienen audio y los personajes muestran una tabla para comunicarse de manera escrita. En la figura 1 se muestran las animaciones.

\section{Animación}

ANIMACIÓN 1

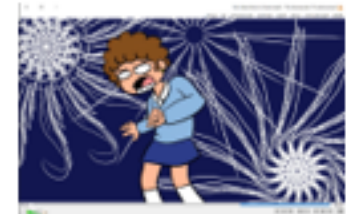

ANIMACIÓN 2

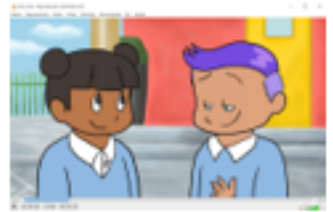

ANIMACIÓN 3

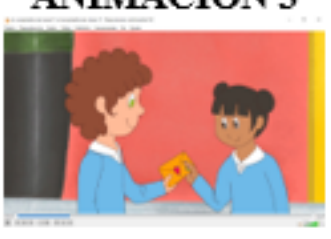

Figura 1. Animaciones 2D para introducir cada tema

\section{¿Qué introduce?}

Animación de bienvenida. Narra la llegada de Camila a la escuela, su primer encuentro con los compañeros y la forma en la que es recibida. Se muestra su frustración cuando Juana, una compañera empieza a hablarle y no entiende nada, por lo que inician a hablar a través de un pizarrón utilizando español.

Se introduce los saludos. La amiga de Camila le presenta a un nuevo compañero que se asusta al ver que no lo escucha, así que inicia la plática a través del pizarrón y a partir de ahí inicia una gran amistad que los marcará en las siguientes aventuras.

El cumpleaños de Juana. Juana, la amiga de Camila celebrará su cumpleaños y la invita a su casa. Durante el festejo juegan y rompen la piñata. En la animación se observan fotos que se han ido tomando durante el festejo, haciendo alusión a vocabulario como: pastel, piñata, cumpleaños, etc.

\section{Fuente: Elaboración propia}

Después de cada proyección introductoria, se llevaron a cabo actividades creadas por el investigador con diferentes herramientas digitales (Flippity, Ardora, Classtools). Para ello, se les solicitó a los profesores que trabajaran con las mencionadas actividades apoyando a los alumnos por si surgían inquietudes. Los enlaces o ligas a las actividades se proveyeron a través de Google Pages.

Al inicio y final de las actividades, los alumnos tenían acceso a un video donde el intérprete explicaba en LSM los temas. A medida que se iba avanzando en la materia, cada alumno podía conocer su progreso y cerrar con un formulario de preguntas enviadas al correo del profesor. 


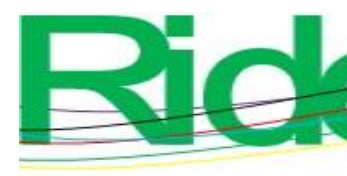

Revista Iberoamericana para la Investigación y el Desarrollo Educativo

ISSN 2007 - 7467

La tercera fase estuvo constituida por ciclos iterativos para probar y depurar las soluciones en la práctica. Es importante resaltar que cada unidad representó una iteración, es decir, la repetición del proceso de enseñanza reflejado en unidades de aprendizaje, lo cual permitió perfeccionar el tipo de actividades desarrolladas. En cada una de las iteraciones se planeó el tema, se desarrolló tanto en el colegio como en la plataforma, se evaluó el uso de actividades en la plataforma y se comenzó una nueva iteración.

Finalmente, la cuarta fase se dedicó a la reflexión en torno al diseño y mejora de la implementación de las soluciones. Para implementar la enseñanza del español se consideró la hipótesis conocida como Sapir-Whorf; según esta, el lenguaje usado por una persona influye en su interpretación del mundo y, por tanto, en su conducta (Zamudio Mesa, 2010), de ahí que una de las preocupaciones más grandes sea la ausencia de grafía en la LSM, por lo que esta sería una novedad para el alumno. El uso de videos con LSM acompañados de subtítulos en español sirvió para este propósito.

\section{Resultados}

La inclusión observada en el grupo en general fue una experiencia positiva, pues los estudiantes sordos, así como los alumnos oyentes interactuaron de forma natural durante los recreos sin necesidad de un intérprete. El uso de herramientas tecnológicas favoreció el aprendizaje de la segunda lengua y la interacción escrita, especialmente con profesores, debido a que el resto de estudiantes oyentes se familiarizó con el uso de LSM y gran parte de la interacción entre estudiantes fue mediante señas.

Durante la primera fase se observaron diferencias en cuanto a la procedencia de los estudiantes. Aquellos procedentes de otro colegio del sector público evidenciaron el uso de señas básicas y algunas inventadas o señalizadas, así como la configuración manual incorrecta y mayor uso de español signado. Asimismo, y a pesar del uso del deletreo, los niños no conocían el orden del alfabeto. En cuanto a la interacción con el medio, los estudiantes desconocían las normas y convencionalismos sociales en un entorno escolar, es decir, no sabían cómo formarse, el orden en clase y el seguimiento de las instrucciones. En lo concerniente a la interacción con otros compañeros, se mostraron abiertos aunque con dificultad en la comunicación.

Los alumnos que tenían su primer contacto con un colegio desconocían la LSM, así como las imágenes y los gestos; sin embargo, se observó una socialización funcional 


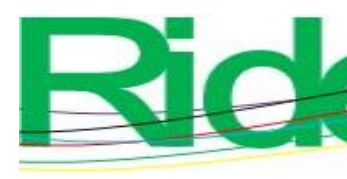

\section{Revista Iberoamericana para la Investigación y el Desarrollo Educativo ISSN 2007 - 7467}

respetando las reglas, siguiendo las instrucciones a través de la imitación y con apertura a recibir nueva información. En cuanto a la socialización, tuvieron buena disposición, pero de igual manera dificultad en la comunicación.

Las observaciones realizadas evidencian que a pesar de que cognitivamente los niños se encuentran en condiciones para el aprendizaje, en casa el reforzamiento sobre los temas es prácticamente inexistente debido a la limitante comunicacional. Por ello, uno de los factores determinantes para el éxito del aprendizaje se halla en la participación de los padres de familia.

Por otra parte, se realizaron entrevistas semiestructuradas para determinar cómo estaba sucediendo la inclusión en el colegio. Para analizar esta información se agruparon los datos en cuatro temáticas recurrentes e interrelacionadas:

- "Inclusión educativa" (IE): Debido a que el colegio ha adoptado un sistema de inclusión educativa, fue recurrente el tema de "niños sordos con oyentes", "los niños que no oyen que están en el salón”, "los maestros que usan las manos para decir las cosas", "la atención a todos los alumnos", etc.

- La segunda categoría estaba enfocada en el concepto de "identidad sorda" (IS): Los participantes hacían énfasis en diferencias entre sordos y oyentes: "los sordos", "la lengua de señas", "el intérprete", "los cantos en lengua de señas".

- La tercera categoría se determinó como "dinámica de grupo de clase” (DG): Esta se vincula con las actividades desarrolladas como grupo sin diferenciar si son sordos u oyentes: "cuando jugamos", "todos participamos", "nadie sabe la respuesta", "nos ayudan", etc.

- Finalmente, la cuarta categoría "relaciones docente-estudiante-colegio-familia" (R): Algunos comentarios asociados a esta fueron: "mis maestros nos ayudan", "la maestra nos dice", "mi hijo pide ayuda en casa", "la maestra nos envía mensajes", "le pedimos información a la maestra", etc.

Durante la fase 3 se observó mayor dificultad para el uso de las herramientas en los padres de familia. Los estudiantes no tuvieron problemas, siempre y cuando se encontraran con su profesora. El uso en casa no tuvo éxito debido a que la mayoría de los estudiantes no tenían acceso a un dispositivo con internet.

En el transcurso de la primera observación, se les permitió indagar en la página sin darles ninguna instrucción. Esto generó mucha confusión en los estudiantes para entrar a la 


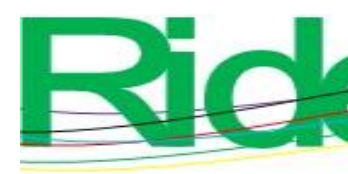

\section{Revista Iberoamericana para la Investigación y el Desarrollo Educativo ISSN 2007 - 7467}

página. Además, fueron evidentes sus preferencias por observar las cosas más llamativas, por lo que en principio se saltaron los videos.

En la segunda observación se hizo un acompañamiento durante cada actividad. Se notó preferencia por los videos de explicación de los profesores y la caricatura de bienvenida de cada sesión. El cuento Camila quiere una mascota fue muy interesante para todos y se sintieron identificados con las situaciones representadas. En general faltó tiempo para poder interactuar con todas las herramientas.

Los videos mostrados en la bienvenida de cada unidad resultaron relevantes para darles a conocer a los estudiantes la forma de incluirse en grupos de personas oyentes. Estos videos también facilitaron la introducción a los temas y favorecieron los aprendizajes esperados.

Con las observaciones se determinó que la herramienta tenía algunos elementos en los cuales el estudiante se mostraba autónomo, mientras que en otros debían ser dirigidos por el profesor.

En cuanto al uso de la herramienta digital en casa, se presentaron dificultades, especialmente por el estrato socioeconómico de algunas familias, las cuales no contaban con una computadora en casa o internet. Por dicho motivo, se facilitaron espacios en el centro de cómputo con los estudiantes y sus tutores.

\section{Discusión}

Durante la fase de exploración y proceso de revisión de la bibliografía se fueron forjando las ideas principales de esta investigación. Se analizaron diferentes métodos de enseñanza de L2 (Lankshear y Knobel, 2008; Lissi, Svartholm y González, 2012; Navarro, 2010) para los estudiantes sordos y su concordancia con el MEM. En este sentido, se apreció que la discrepancia fundamental reside en la forma de comunicación, y no en las técnicas o métodos. Sin embargo, se identificó una preferencia para enseñar español a sordos basados en el enfoque oralista, así como en el bilingüe-bicultural, los cuales son antagónicos, ya que el primero es médico regenerativo y el segundo se basa en la pertenencia a una cultura (Cole y Flexer, 2019; Goldin-Meadow y Mayberry, 2001; Lissi, Svartholm y González, 2012). También se observó un enfoque bimodal, más utilizado en países europeos, el cual combina el uso de la voz y la LSM (Alcina Madueño, 2015, Sepúlveda, 2008). El diseño presentado se basó en el enfoque bilingüe-bicultural. 


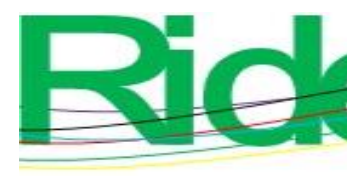

Revista Iberoamericana para la Investigación y el Desarrollo Educativo ISSN 2007 - 7467

Por otra parte, se evaluó cognitivamente a los alumnos sordos con sus pares oyentes, y no se observaron contrastes fundamentales en su capacidad para aprender. No obstante, algunas investigaciones (Goldin Meadow y Mayberry, 2001; Hurford, 1991; Lieberman, Hatrak y Mayberry, 2014) sobre el aprendizaje de las personas sordas han establecido que los mismos hemisferios utilizados por las personas oyentes cuando se procesa el lenguaje se activan en las personas sordas cuando utilizan la representación gráfica y la LSM.

En la actualidad, aunque se tuvieron presentes las políticas internacionales, nacionales y locales (Brunot, 2019; García, 2018; Sánchez Regalado, 2012; SEP, 2017, 2018) que permiten la apertura de espacios educativos como el que se realizó en la investigación, se advierte que aún es necesaria la declaración oficial de que las personas sordas pueden recibir educación en su propia lengua. Esto obligaría a nuestro sistema educativo a ajustar los textos a la LSM o a contratar a intérpretes educativos de LSM, para los cuales la preparación aún es incipiente. Mientras tanto, el uso de tecnologías de la información para mejorar la educación de las personas sordas en una L2 puede ser un primer esfuerzo para ofrecer más espacios educativos de una manera menos costosa.

Como en el estado de Querétaro no existe otro colegio de nivel básico con el modelo bilingüe-bicultural propuesto, su selección consistió elementalmente en la aceptación del modelo, el cual es costoso porque requiere — además del profesor- de la contratación de un intérprete educativo de LSM por grado. Consideramos de gran importancia explicar la función educativa desde el marco marista, que en cuanto al proyecto de las personas sordas no tiene una pretensión económica, sino un fundamento apoyado en su ideario y en sus bases religiosas que pregonan la misericordia y el carisma para escuchar a los niños y a los jóvenes, especialmente a aquellos más desfavorecidos.

La determinación de los principios de diseño de una herramienta digital para la enseñanza de español como L2 se pueden aplicar en cualquier otra materia, tomando en cuenta que la LSM siempre tendrá un papel fundamental para la enseñanza acompañada del español (Cobb et al., 2003; Kelly, 2003; Wang y Hannafin, 2005).

El entorno digital que acoge diferentes herramientas tecnológicas, como menciona Fontán (2004), es muy amplio, por lo que el profesor del grupo ya no es el único responsable del ambiente de aprendizaje, sino que entran en acción otros agentes, como los directores, las familias, los profesionales y las propias personas sordas a partir de sus experiencias. En consecuencia, la tecnología educativa puede ser una herramienta poderosa para mejorar los 


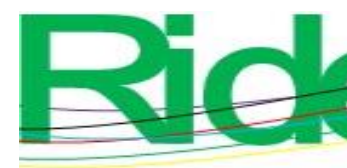

Revista Iberoamericana para la Investigación y el Desarrollo Educativo

ISSN 2007 - 7467

resultados de aprendizaje de los estudiantes (Cacheiro et al., 2016; Martínez et al., 2018). Esta puede ayudar a los docentes a ampliar los horizontes de los estudiantes y permitirles explorar los límites de su libertad. De igual manera puede agregar valor a la lección.

Sobre las familias, estas mostraron interés por conocer la herramienta, aunque vale acotar que la mayoría tiene dificultad para utilizar computadoras con sus hijos, ya que en general los estudiantes provienen de hogares cuyos padres estudiaron hasta el nivel de bachillerato.

Para los niños fue llamativo el empleo de las tecnologías para los aprendizajes, sobre todo porque vieron plasmadas sus historias en la herramienta y se sentían identificados especialmente en el cuento y en la caricatura animada.

Se puede decir que el uso de tecnología es aceptado siempre y cuando se tenga la herramienta a la mano, los juegos sean interesantes y no represente un reto imposible para los estudiantes.

\section{Conclusiones}

Para crear una herramienta digital dirigida a estudiantes sordos las actividades se deben basar en el juego y la autoevaluación, la cual se debe ir desarrollando a medida que se ejecuta cada actividad, sin sacrificar la parte lúdica. En tal sentido, el uso de herramientas digitales para la enseñanza de español como L2 a niños sordos resulta ser una opción para complementar los aprendizajes vistos en el aula. Sin embargo, se debe comprender que los niños sordos desarrollan el lenguaje de manera tardía, y que su L1, al ser ágrafa, constituye un reto para la enseñanza de la L1.

Para atender esta situación, los juegos en línea prometen generar una afición en los niños cuando los objetivos son alcanzables y divertidos; por tanto, el acompañamiento a estos estudiantes (bien sea por el profesor o sus padres) ayuda a determinar si la actividad cumplió el objetivo. A la hora de desarrollar contenidos digitales, es necesario contemplar a quién va dirigido y cuáles son las características del alumnado; pensar en su globalidad, diseñarlos como un recurso integrado a un proceso de aprendizaje que abarca otros temas. En pocas palabras, se debe actuar con mucha didáctica para los niños sean capaces de entender, razonar y llevar a cabo aquello que se intenta transmitir. Por eso, el material para educandos menores de edad debe ser sencillo de comprender, con objetivos claros, divertido y visual. Exponer a 


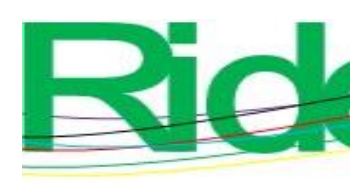

Revista Iberoamericana para la Investigación y el Desarrollo Educativo

ISSN 2007 - 7467

los estudiantes al español desde muy temprano es el ideal debido a la plasticidad cerebral que con el tiempo disminuye.

También se debe tener presente que no existe el método perfecto para enseñar a un estudiante sordo, aunque sí se deben considerar primordialmente recursos visuales y kinestésicos a la hora de aprender, sabiendo que en ciertos casos estos podían incluso representar un "ruido" que interrumpiría el aprendizaje.

Otra determinante a la hora de aprender una L2 tiene su razón en que se tenga una base sólida en la L1, es decir, los individuos que tienen mejores habilidades en la LSM tienen mayores posibilidades de no confundirse cuando aprenden español.

El aprendizaje es más significativo cuando resulta relevante culturalmente. Esto significa que cuando los estudiantes sordos se sienten como integrantes de una comunidad sorda, los elementos que se integren a la enseñanza desde su comunidad resultarán con más posibilidades de ser aprendidos.

Por otra parte, se debe prever que los actos de leer y escribir exigen diferentes procesos simultáneos. Cuando se lee se deben decodificar las palabras y entender lo que significan. Para las personas sordas las conjunciones, los nexos, los artículos, entre otros, son inexistentes en su diálogo de LSM. Todo lo anterior representa un gran reto a la hora de enseñar. Por tanto, la tecnología no es ni será nunca por sí misma una solución infalible para la enseñanza de la lengua o de cualquier otro tema. Si bien es cierto que la tecnología acompañada de un buen diseño educativo puede favorecer en mucho la enseñanza de la L2, no es lo único que hay que utilizar.

Es un hecho que los estudiantes sordos se sienten parte de la comunidad escolar; no obstante, también es lógico que prefieran reunirse con sus pares sordos debido a la mejor comunicación que pueden establecer con ellos, aunque a medida que avanzan de grado en el colegio se observa más inclusión con los demás, sobre todo en los recreos.

Por ello, si se logra establecer un ambiente escolar de mayor naturalidad para ellos, existirán mejores posibilidades para que la inclusión sea exitosa. El hecho está en entender que siendo todos somos diferentes, nos aceptamos con nuestras virtudes e imperfecciones.

Finalmente, el siguiente paso en la investigación es llevar a cabo el proceso de inclusión en todos los grados escolares, lo cual implica gestionar recursos económicos para las siguientes producciones, involucrar a todos los profesores y asociarse con diferentes instituciones interesadas. 


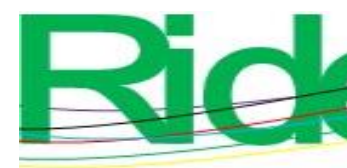

Revista Iberoamericana para la Investigación y el Desarrollo Educativo

ISSN 2007 - 7467

\section{Referencias}

Alcina Madueño, A. (2015). Las lenguas de signos en la formación de los maestros de sordos en España. Una visión histórica. Revista de Educación, 349, 437-449.

Armstrong, C., Armstrong, D. and Spandagou, I. (2010). Inclusive education. International policy \& practice. Journal of Research in Special Education Needs, 10 (3), 256-271.

Bell, P., Hoadley, C. M. and Linn, M. C. (2004). Design-based research. In M. C. Linn, E. A. Davis and P. Bell (eds.). Internet environments for science education (pp. 73-88). Mahwah, New Jersey, Lawrence Erlbaum Associates.

Bisquerra, R. (2016). Metodología de la investigación educativa (pp. 321-357) Madrid: La Muralla, S.A.

Bonilla, M. F., Fernández, J. y Vázquez, M. A. (2019). Diagnóstico del trabajo colaborativo en un centro escolar como indicador de inclusión educativa. RIDE Revista Iberoamericana para la Investigación y el Desarrollo Educativo, 10(19). Doi: https://doi.org/10.23913/ride.v10i19.518

Booth, T. y Ainscow, M. (2002). Guía para la evaluación y mejora de la educación inclusiva. Index for Inclusion. Bristol: UNESCO.

Brennan, M. (1975). Can deaf children acquire language? An evaluation of linguistic principles in deaf education. American Annals of the Deaf, 120 (5), 463-479.

Fridman, B. (2009). De sordos hablantes, semilingües y señantes. LynX Panorámica de Estudios Lingüísticos, 8, 93-126.

Brunot, S. (2019). Inclusión de niños de 6 a 7 años con necesidades educativas especiales (discapacidades sensorial, mental e intelectual) en una escuela primaria con Unidad de Servicio de Apoyo a la Educación Regular, en San Luis Potosí, México. Revista de El Colegio de San Luis, 9(18), 69-109. Doi: https://dx.doi.org/10.21696/rcs19182019815

Cacheiro, M. L. Sánchez, C. y González, J. M. (coords.). (2016). Recursos tecnológicos en contextos educativos. Madrid: Universidad Nacional de Educación a Distancia.

Cobb, P., Confrey, J., DiSessa, A., Lehrer, R. and Schauble, L. (2003). Design experiments in educational research. Educational Researcher, 32(1), 9-13.

Cole, E. B. and Flexer, C. (2019). Children with hearing loss: Developing listening and talking, birth to six. Plural Publishing. 


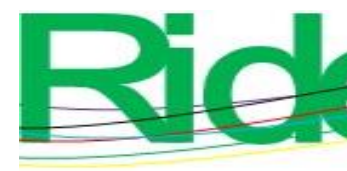

Revista Iberoamericana para la
Investigación y el Desarrollo Educativo
ISSN $2007-7467$

Fontán, M.T. (2004). Evaluar a través de internet. Pixel-Bit. Revista de Medios y Educación, 24, 79-88.

García, I. (2018). La educación inclusiva en la Reforma Educativa de México. Revista Nacional e Internacional de Educación Inclusiva, 11(2), 49-62.

Gertz, G. and Boudreault, P. (2016). The SAGE deaf studies encyclopedia. Doi: http://dx.doi.org/10.4135/9781483346489

Gibelli, T. (2014). La investigación basada en diseño para el estudio de una innovación en educación superior que promueve la autorregulación del aprendizaje utilizando TIC. En Congreso Iberoamericano de Ciencia, Tecnología, Innovación y Educación (116).

Goldin-Meadow, S. and Mayberry, R. I. (2001). How do profoundly deaf children learn to read? Learning Disabilities Research \& Practice, 16(4), 222-229.

Gros, B. (2007). Digital games in education: The design of games-based learning environments. Journal of Research on Technology in Education, 40(1), 23-38.

Hurford, J. R. (1991). The evolution of the critical period for language acquisition. Cognition, 40(3), 159-201.

Kelly, A. E. (2003). Theme issue:The role of design in educational research. Educational Researcher, 32(1), 3-4.

Lankshear, C. J. and Knobel, M. (2008). Introduction: digital literacies: concepts, policies and practices. Peter Lang Publishing.

Lieberman, A. M., Hatrak, M. and Mayberry, R. I. (2014). Learning to look for language: Development of joint attention in young deaf children. Language Learning and Development, 10(1), 19-35.

Lissi, M. R., Svartholm, K. y González, M. (2012). El enfoque bilingüe en la educación de sordos: sus implicancias para la enseñanza y aprendizaje de la lengua escrita. Estudios Pedagógicos (Valdivia), 38(2), 299-320.

Martínez, S., Gutiérrez, J. J. y Fernández, B. (2018). Percepciones y uso de las TIC en las aulas inclusivas. Un estudio de caso. EDMETIC, Revista de Educación Mediática, 7(1), 87-106. Doi: https://doi.org/10.21071/edmetic.v7i1.10132

McKenney, S. E. and Reeves, T. C. (2012). Conducting educational research design: what, why and how (pp. 27-29). London: Routledge. 


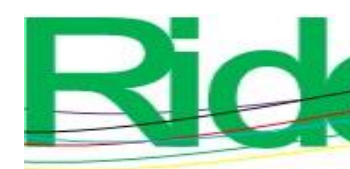

Revista Iberoamericana para la Investigación y el Desarrollo Educativo

ISSN 2007 - 7467

McKenney, S., van den Akker, J. and Nieveen, N. (2006). Design research from the curriculum perspective. In J. Van den Akker, K. Gravemeijer, S. McKenney and N. Nieveen (eds.), Educational design research (pp. 67-90). London: Routledge.

Mitchell, R. E. (2004). Chasing the mythical ten percent: Parental hearing status of deaf and hard of hearing students in the United States. Sign language studies, 4(2), 138-163.

Moores, D. F. (1990). Educational and developmental aspects of deafness. Gallaudet University Press.

Navarro, B. (2010). Adquisición de la primera y segunda lengua en aprendientes en edad infantil y adulta. Revista Semestral de Iniciación a la Investigación en Filología, 2, 115-128.

Reeves, T. C. (2006). Design research from the technology perspective. In JV Akker, K. Gravemeijer, S. McKenney ve N. Nieveen (eds.), Educational design research içinde (86-109).

Romero, S. y García, I. (2013). Educación especial en México. Desafíos de la educación inclusiva. Revista Latinoamericana de Educación Inclusiva, 7(1), 77-91.

Ruiz de Chávez, E., Robles, J., Ramírez, H., Jiménez, H. y Meza. (2016). Provincia Marista de México Central: modelo educativo marista. México: EPPE.

Sánchez Regalado, N. P. (2012). El currículo de la educación básica en México: un proyecto educativo flexible para la atención a la diversidad y el fortalecimiento de la sociedad democrática. Revista Iberoamericana sobre Calidad, Eficiencia y Cambio en Educación, 10(4), 150-163.

Secretaría de Educación Pública (SEP) (2015). Evaluación de diseño. Programa para la inclusión y la equidad educativa (pp. 1-42). México: SEP.

Secretaría de Educación Pública (SEP) (2016). El modelo educativo 2016 (64-86) México: SEP.

Secretaría de Educación Pública (SEP) (2017). Ruta para la implementación del modelo educativo (80-110). México: SEP.

Sepúlveda, C. B. (2008). Lenguaje y educación en niños sordos: encuentros y desencuentros. REXE. Revista de Estudios y Experiencias en Educación, 14, 105-114.

Skotara, N., Salden, U., Kügow, M., Hänel-Faulhaber, B. and Röder, B. (2012). The influence of language deprivation in early childhood on L2 processing: An ERP 

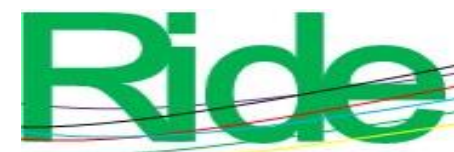

Revista Iberoamericana para la Investigación y el Desarrollo Educativo

ISSN 2007 - 7467

comparison of deaf native signers and deaf signers with a delayed language acquisition. BMC Neuroscience, 13(1), 44.

Stokoe, W. C. (2005). Sign language structure: An outline of the visual communication systems of the American deaf. Journal of Deaf Studies and Deaf Education, 10 (1), 3-37.

Susskind, R. y Susskind, D. (2017). The Future of the Professions: How Technology Will Transform the Work of Human Experts. Administrative Science Quarterly, 62 (4), 34-42.

Unicef (2017). Unicef México. Informe Anual 2017. Recuperado de https://www.unicef.org.mx/Informe2017/Informe-Anual-2017.pdf

Wang, F. and Hannafin, M. J. (2005). Design-based research and technology-enhanced learning environments. Educational technology research and development, 53 (4), 523.

Zamudio Mesa, C. M. (2010). Las consecuencias de la escritura alfabética en la teoría lingüística. México: El Colegio de México. 


\begin{tabular}{|l|l|}
\hline Rol de Contribución & Autor (es) \\
\hline Conceptualización & Paloma Trejo Muñoz \\
\hline Metodología & Paloma Trejo Muñoz \\
\hline Software & Paloma Trejo Muñoz \\
\hline Validación & Paloma Trejo Muñoz/Sandra Martínez Pérez «igual» \\
\hline Análisis Formal & Paloma Trejo Muñoz /Sandra Martínez Pérez «igual» \\
\hline Investigación & Paloma Trejo Muñoz \\
\hline Recursos & Rodrigo Espinosa Larracoechea \\
\hline Curación de datos & Paloma Trejo Muñoz/Sandra Martínez Pérez «igual» \\
\hline $\begin{array}{l}\text { Escritura - Preparación del } \\
\text { borrador original }\end{array}$ & Paloma Trejo Muñoz \\
\hline $\begin{array}{l}\text { Escritura - Revisión y } \\
\text { edición }\end{array}$ & Sandra Martínez Pérez \\
\hline Visualización & Paloma Trejo Muñoz \\
\hline Supervisión & Sandra Martínez Pérez \\
\hline Administración de & Paloma Trejo Muñoz \\
\hline Adquisición de fondos & \\
\hline
\end{tabular}

